\title{
Solutions to unsolved problems on the minimal energies of two classes of trees
}

\author{
Yongqiang Bai, Hongping $\mathrm{Ma}^{*}$ \\ School of Mathematics and Statistics, Jiangsu Normal University, \\ Xuzhou 221116, China
}

\begin{abstract}
The energy of a graph is defined as the sum of the absolute values of all eigenvalues of the graph. Let $\mathbb{T}_{n, p}, \mathcal{T}_{n, d}$ be the set of all trees of order $n$ with $p$ pendent vertices, diameter $d$, respectively. In this paper, we completely characterize the trees with second-minimal and third-minimal energy in $\mathbb{T}_{n, p}$ $\left(\mathcal{T}_{n, d}\right.$, respectively) for $4 \leq p \leq n-9$ (10 $\leq d \leq n-3$, respectively), which solves the problems left in [20].
\end{abstract}

Keywords: Minimal energy; Tree; Pendent vertex; Diameter

\section{Introduction}

Let $G$ be a simple graph with $n$ vertices, and $\lambda_{1}, \lambda_{2}, \ldots, \lambda_{n}$ be the eigenvalues of its adjacency matrix. Then the energy of $G$ is defined as

$$
E=E(G)=\sum_{i=1}^{n}\left|\lambda_{i}\right|
$$

Let $G$ be an acyclic graph of order $n$. Then $E(G)$ can be expressed as the Coulson integral formula [11]

$$
E(G)=\frac{2}{\pi} \int_{0}^{+\infty} \frac{1}{x^{2}} \log \left[\sum_{k=0}^{\left\lfloor\frac{n}{2}\right\rfloor} m(G, k) x^{2 k}\right] d x
$$

where $m(G, k)$ denotes the number of $k$-matchings in $G$ and $m(G, 0)=1$. Follow [15], denote

$$
m^{+}(G, x)=\sum_{k=0}^{\left\lfloor\frac{n}{2}\right\rfloor} m(G, k) x^{2 k} .
$$

Then for a tree $T$ with $n$ vertices, we have

$$
E(T)=\frac{2}{\pi} \int_{0}^{+\infty} \frac{1}{x^{2}} \log m^{+}(T, x) d x .
$$

\footnotetext{
${ }^{*}$ Corresponding author. E-mail addresses: bmbai@163.com (Y. Bai), hpma@163.com (H. Ma)
} 
Since the energy of a graph can be used to approximate the total $\pi$-electron energy of the molecular, it has been intensively studied. For details on graph energy, we refer the readers to the book [18] and reviews $[8,10]$. One of the fundamental question that is encountered in the study of graph energy is which graphs (from a given class) have minimal and maximal energies. A large number of papers were published on such extremal problems, see Chapter 7 in [18] and some recent papers $[9,12,13,15$ 17,19-30]. There are many other kinds of graph energies, such as matching energy [1, 2, 3], Laplacian energy [4], distance energy [5], Randić energy [6, 14], incidence energy [6], etc.

For terminology and notation not defined here, we refer to [20]. A caterpillar is a tree in which a removal of all pendent vertices makes a path. Let $T\left(n, d ; n_{1}, n_{2}, \ldots, n_{d-1}\right)$ be a caterpillar obtained from a path $v_{0} v_{1} \cdots v_{d}$ by adding $n_{i}\left(n_{i} \geq 0\right)$ pendent edges to $v_{i}(i=1, \ldots, d-1)$. For $1 \leq i \leq d-1$, denote $T_{n, d, i}=T\left(n, d ; 0, \ldots, 0, n_{i}=\right.$ $n-d-1,0, \ldots, 0)$.

Gutman [7] proved that the star $S_{n}$ and the path $P_{n}$ have minimal and maximal energy among all trees of order $n$, respectively. Let $\mathcal{T}_{n, d}$ be the set of all trees with $n$ vertices and diameter $d$, where $2 \leq d \leq n-1$. The trees with minimal and secondminimal energies in $\mathcal{T}_{n, d}$ have been considered by $[27,30,16,17,26,24]$. Let $\mathbb{T}_{n, p}$ be the set of all trees of order $n$ with $p$ pendent vertices, where $2 \leq p \leq n-1$. The tree with minimal energy in $\mathbb{T}_{n, p}$ has been determined by [29] and [28] independently. By the results in [27], [29] and [28], the trees with minimal energy in $\mathcal{T}_{n, d}$ and $\mathbb{T}_{n, n-d+1}$ are the same for all $2 \leq d \leq n-1$. In [20], one of the present authors gave the further relations on the ordering of trees by minimal energies between $\mathcal{T}_{n, d}$ and $\mathbb{T}_{n, n-d+1}$, and completely characterized the trees with second-minimal and third-minimal energy in $\mathbb{T}_{n, p}\left(\mathcal{T}_{n, d}\right.$, respectively) except for the case $4 \leq p \leq n-9(10 \leq d \leq n-3$, respectively). By [20], the trees with second-minimal and third-minimal energy in $\mathbb{T}_{n, p}$ and $\mathcal{T}_{n, n-p+1}$ are the same, respectively, for $4 \leq p \leq n-9$, and the results on $\mathbb{T}_{n, p}$ are the following:

Theorem 1.1. [20] Suppose that $4 \leq p \leq n-9$. Then the second-minimal energy tree in $\mathbb{T}_{n, p}$ is one of the two trees $T_{n, n-p+1,3}$ and $T(n, n-p+1 ; p-3,0, \ldots, 0,1)$.

Theorem 1.2. [20] Suppose that $4 \leq p \leq n-9$. If $E\left(T_{n, n-p+1,3}\right)>E(T(n, n-p+$ $1 ; p-3,0, \ldots, 0,1))$, then $T_{n, n-p+1,3}$ is the unique tree with third-minimal energy in $\mathbb{T}_{n, p} ;$ If $E\left(T_{n, n-p+1,3}\right)<E(T(n, n-p+1 ; p-3,0, \ldots, 0,1))$, then the third-minimal energy tree in $\mathbb{T}_{n, p}$ is one of the two trees $T_{n, n-p+1,5}$ and $T(n, n-p+1 ; p-3,0, \ldots, 0,1)$.

From Theorems 1.1 and 1.2, one can see that for $4 \leq p \leq n-9$, the author of [20] could not determine the unique extremal graph, respectively. In this paper, we will use the Coulson integral formula to solve the above unsolved problems. We only state our results on $\mathbb{T}_{n, p}$ instead of $\mathcal{T}_{n, d}$. 
The rest of this paper is organized as follows. In Section 2, we give some preliminary results. In Section 3, we characterize the trees with second-minimal energy in $\mathbb{T}_{n, p}$ for $4 \leq p \leq n-9$. In Section 4 , we determine the trees with third-minimal energy in $\mathbb{T}_{n, p}$ for $4 \leq p \leq n-9$.

\section{Preliminaries}

The following five results on properties of $m^{+}(G, x)$ appeared in [15].

Lemma 2.1. Let $v$ be a vertex of graph $G$ and $N(v)=\left\{v_{1}, v_{2}, \ldots, v_{j}\right\}$ the set of all neighbors of $v$ in $G$. Then

$$
m^{+}(G, x)=m^{+}(G-v, x)+x^{2} \sum_{v_{i} \in N(v)} m^{+}\left(G-v-v_{i}, x\right) .
$$

Lemma 2.2. Let $P_{t}$ be the path on $t$ vertices. Then

$$
\begin{aligned}
& \text { (1) } m^{+}\left(P_{t}, x\right)=m^{+}\left(P_{t-1}, x\right)+x^{2} m^{+}\left(P_{t-2}, x\right) \text {, for any } t \geq 1 \text {, } \\
& \text { (2) } m^{+}\left(P_{t}, x\right)=\left(1+x^{2}\right) m^{+}\left(P_{t-2}, x\right)+x^{2} m^{+}\left(P_{t-3}, x\right) \text {, for any } t \geq 2 \text {. }
\end{aligned}
$$

The initials are $m^{+}\left(P_{0}, x\right)=m^{+}\left(P_{1}, x\right)=1$, and we define $m^{+}\left(P_{-1}, x\right)=0$.

Corollary 2.3. Suppose $t \geq 1$. Then for any real number $x$,

$$
m^{+}\left(P_{t-1}, x\right) \leq m^{+}\left(P_{t}, x\right) \leq\left(1+x^{2}\right) m^{+}\left(P_{t-1}, x\right) .
$$

Lemma 2.4. For $t \geq-1$, the polynomial $m^{+}\left(P_{t}, x\right)$ has the following form

$$
m^{+}\left(P_{t}, x\right)=\frac{1}{\sqrt{1+4 x^{2}}}\left(\lambda_{1}^{t+1}-\lambda_{2}^{t+1}\right)
$$

where $\lambda_{1}=\frac{1+\sqrt{1+4 x^{2}}}{2}$ and $\lambda_{2}=\frac{1-\sqrt{1+4 x^{2}}}{2}$.

Lemma 2.5. Suppose $t \geq 0$. If $t$ is even, then

$$
\frac{2}{1+\sqrt{1+4 x^{2}}} \leq \frac{m^{+}\left(P_{t}, x\right)}{m^{+}\left(P_{t+1}, x\right)} \leq 1 .
$$

If $t$ is odd, then

$$
\frac{1}{1+x^{2}} \leq \frac{m^{+}\left(P_{t}, x\right)}{m^{+}\left(P_{t+1}, x\right)} \leq \frac{2}{1+\sqrt{1+4 x^{2}}} .
$$

The following result on real analysis is needed in the next sections.

Lemma 2.6. [31] For any real number $X>-1$, we have

$$
\frac{X}{1+X} \leq \log (1+X) \leq X .
$$




\section{The trees with second-minimal energy in $\mathbb{T}_{n, p}$}

In this section, we will characterize the trees with second-minimal energy in $\mathbb{T}_{n, p}$ for $4 \leq p \leq n-9$. For convenience, we define $T_{1}=T(n, n-p+1 ; p-3,0, \ldots, 0,1)$, $T_{2}=T_{n, n-p+1,3}$ and $T_{3}=T_{n, n-p+1,5}$.

By Lemmas 2.1 and 2.2, we can easily get the following result.

Lemma 3.1. Suppose that $4 \leq p \leq n-9$. Then we have

$$
\begin{aligned}
& m^{+}\left(T_{1}, x\right)=A_{1} m^{+}\left(P_{n-p-2}, x\right)+A_{2} m^{+}\left(P_{n-p-3}, x\right) \\
& m^{+}\left(T_{2}, x\right)=B_{1} m^{+}\left(P_{n-p-2}, x\right)+B_{2} m^{+}\left(P_{n-p-3}, x\right)
\end{aligned}
$$

where $A_{1}=1+(p+1) x^{2}+2(p-2) x^{4}, A_{2}=x^{2}+p x^{4}, B_{1}=1+(p+1) x^{2}+(2 p-3) x^{4}$, and $B_{2}=x^{2}+2 x^{4}$.

Theorem 3.2. (i) If $5 \leq p \leq n-9$, then $T_{2}$ is the unique tree with second-minimal energy in $\mathbb{T}_{n, p}$.

(ii) $T_{2}$ if $n=13, T_{1}$ if $n \geq 14$ is the unique tree with second-minimal energy in $\mathbb{T}_{n, 4}$

Proof. From Eq. (1), we have

$$
\begin{aligned}
E\left(T_{1}\right)-E\left(T_{2}\right) & =\frac{2}{\pi} \int_{0}^{+\infty} \frac{1}{x^{2}} \log \frac{m^{+}\left(T_{1}, x\right)}{m^{+}\left(T_{2}, x\right)} d x \\
& =\frac{2}{\pi} \int_{0}^{+\infty} \frac{1}{x^{2}} \log \left(1+\frac{m^{+}\left(T_{1}, x\right)-m^{+}\left(T_{2}, x\right)}{m^{+}\left(T_{2}, x\right)}\right) d x
\end{aligned}
$$

(i) Suppose that $5 \leq p \leq n-9$. By Lemmas 2.6, 3.1 and Corollary 2.3, we have

$$
\begin{aligned}
& E\left(T_{1}\right)-E\left(T_{2}\right) \\
& \geq \frac{2}{\pi} \int_{0}^{+\infty} \frac{1}{x^{2}} \cdot \frac{m^{+}\left(T_{1}, x\right)-m^{+}\left(T_{2}, x\right)}{m^{+}\left(T_{1}, x\right)} d x \\
& =\frac{2}{\pi} \int_{0}^{+\infty} \frac{-x^{2} m^{+}\left(P_{n-p-2}, x\right)+(p-2) x^{2} m^{+}\left(P_{n-p-3}, x\right)}{A_{1} m^{+}\left(P_{n-p-2}, x\right)+A_{2} m^{+}\left(P_{n-p-3}, x\right)} d x \\
& =\frac{2}{\pi} \int_{0}^{+\infty} \frac{(p-2) x^{2}}{A_{2}+A_{1} \cdot \frac{m^{+}\left(P_{n-p-2}, x\right)}{m^{+}\left(P_{n-p-3}\right)}} d x-\frac{2}{\pi} \int_{0}^{+\infty} \frac{x^{2}}{A_{1}+A_{2} \cdot \frac{m^{+}\left(P_{n-p-3}, x\right)}{m^{+}\left(P_{n-p-2}\right)}} d x \\
& \geq \frac{2}{\pi} \int_{0}^{+\infty} \frac{(p-2) x^{2}}{A_{2}+A_{1} \cdot\left(1+x^{2}\right)} d x-\frac{2}{\pi} \int_{0}^{+\infty} \frac{x^{2}}{A_{1}+A_{2} \cdot \frac{1}{1+x^{2}}} d x \\
& =\frac{2}{\pi} \int_{0}^{+\infty} \frac{\left(p-3-x^{2}\right) x^{2}}{1+(p+3) x^{2}+(4 p-3) x^{4}+2(p-2) x^{6}} d x \\
& \triangleq F(p) .
\end{aligned}
$$


For a fixed $x>0$, let

$$
f(p)=\frac{\left(p-3-x^{2}\right) x^{2}}{1+(p+3) x^{2}+(4 p-3) x^{4}+2(p-2) x^{6}} .
$$

Then we have

$$
f^{\prime}(p)=\frac{x^{2}+6 x^{4}+10 x^{6}+6 x^{8}+2 x^{10}}{\left[1+(p+3) x^{2}+(4 p-3) x^{4}+2(p-2) x^{6}\right]^{2}}>0 .
$$

So $f(p)$ is a strictly increase function of $p$. Since $F(5) \doteq \frac{2}{\pi} \cdot 0.0091327>0$, we can obtain that for $p \geq 5$,

$$
E\left(T_{1}\right)-E\left(T_{2}\right) \geq F(p) \geq F(5)>0 .
$$

(ii) Now suppose that $p=4$. Then by Eq. (2), we have

$$
\begin{aligned}
& E\left(T_{1}\right)-E\left(T_{2}\right) \\
& =\frac{2}{\pi} \int_{0}^{+\infty} \frac{1}{x^{2}} \log \left(1+\frac{2 x^{4}}{B_{2}+B_{1} \cdot \frac{m^{+}\left(P_{n-p-2}, x\right)}{m^{+}\left(P_{n-p-3}, x\right)}}-\frac{x^{4}}{B_{1}+B_{2} \cdot \frac{m^{+}\left(P_{n-p-3}, x\right)}{m^{+}\left(P_{n-p-2}, x\right)}}\right) d x
\end{aligned}
$$

where $B_{1}=1+5 x^{2}+5 x^{4}$ and $B_{2}=x^{2}+2 x^{4}$ by Lemma 3.1 .

We first consider the case that $n$ is even. By Eq. (3) and Lemma 2.5, we have

$$
\begin{aligned}
E\left(T_{1}\right)-E\left(T_{2}\right) & \leq \frac{2}{\pi} \int_{0}^{+\infty} \frac{1}{x^{2}} \log \left(1+\frac{2 x^{4}}{B_{2}+B_{1} \cdot \frac{1+\sqrt{1+4 x^{2}}}{2}}-\frac{x^{4}}{B_{1}+B_{2} \cdot \frac{2}{1+\sqrt{1+4 x^{2}}}}\right) d x \\
& \doteq \frac{2}{\pi} \cdot(-0.018552)<0 .
\end{aligned}
$$

If $n$ is odd, we want to find $n$ and $x$ satisfying that

$$
\frac{m^{+}\left(P_{n-p-3}, x\right)}{m^{+}\left(P_{n-p-2}, x\right)}<\frac{2.01}{1+\sqrt{1+4 x^{2}}}
$$

By Lemma 2.4, it is equivalent to solve

$$
\frac{\lambda_{1}^{n-p-2}-\lambda_{2}^{n-p-2}}{\lambda_{1}^{n-p-1}-\lambda_{2}^{n-p-1}}<\frac{2.01}{2 \lambda_{1}}
$$

which means to solve

$$
\left(\frac{\lambda_{1}}{-\lambda_{2}}\right)^{n-p-2}>\frac{2 \lambda_{1}-2.01 \lambda_{2}}{0.01 \lambda_{1}}
$$

that is,

$$
\left(\frac{1+\sqrt{1+4 x^{2}}}{2 x}\right)^{2(n-p-2)}>401-\frac{402}{1+\sqrt{1+4 x^{2}}}
$$


or equivalently,

$$
2(n-p-2)>\log _{\frac{1+\sqrt{1+4 x^{2}}}{2 x}}\left(401-\frac{402}{1+\sqrt{1+4 x^{2}}}\right) .
$$

Note that for $x \in(0,+\infty), \log _{\frac{1+\sqrt{1+4 x^{2}}}{2 x}}\left(401-\frac{402}{1+\sqrt{1+4 x^{2}}}\right)$ is increasing. Therefore if $x \in(0,19.5]$, then

$\log _{\frac{1+\sqrt{1+4 x^{2}}}{2 x}}\left(401-\frac{402}{1+\sqrt{1+4 x^{2}}}\right) \leq \log _{\frac{1+\sqrt{1+4 \cdot(19.5)^{2}}}{2 \cdot 19.5}}\left(401-\frac{402}{1+\sqrt{1+4 \cdot(19.5)^{2}}}\right)<233$.

Thus when $n \geq 123$ and $x \in(0,19.5]$, Ineq. (4) holds. Hence if $n$ is odd and $n \geq 123$, from Eq. (3) and Lemma 2.5, we have

$$
\begin{aligned}
E\left(T_{1}\right)-E\left(T_{2}\right) & \leq \frac{2}{\pi} \int_{19.5}^{+\infty} \frac{1}{x^{2}} \log \left(1+\frac{2 x^{4}}{B_{2}+B_{1} \cdot 1}-\frac{x^{4}}{B_{1}+B_{2} \cdot 1}\right) d x \\
& +\frac{2}{\pi} \int_{0}^{19.5} \frac{1}{x^{2}} \log \left(1+\frac{2 x^{4}}{B_{2}+B_{1} \cdot \frac{1+\sqrt{1+4 x^{2}}}{2.01}}-\frac{x^{4}}{B_{1}+B_{2} \cdot \frac{2.01}{1+\sqrt{1+4 x^{2}}}}\right) d x, \\
& \doteq \frac{2}{\pi} \cdot\left(-8.1799 \times 10^{-5}\right)<0 .
\end{aligned}
$$

For any odd $n$ with $13 \leq n \leq 121$, by computing the energies of the two graphs directly, we get that $E\left(T_{1}\right)<E\left(T_{2}\right)$ for $n \geq 15$ and $E\left(T_{1}\right)>E\left(T_{2}\right)$ for $n=13$.

The proof is thus complete by Theorem 1.1.

\section{The trees with third-minimal energy in $\mathbb{T}_{n, p}$}

In this section, we will characterize the trees with third-minimal energy in $\mathbb{T}_{n, p}$ for $4 \leq p \leq n-9$. For the case $p=4$, we have the following theorem.

Theorem 4.1. $T_{1}$ if $n=13, T_{2}$ if $n \geq 14$ is the unique tree with third-minimal energy tree in $\mathbb{T}_{n, 4}$.

Proof. By direct computation, we have $E\left(T_{3}\right)>E\left(T_{1}\right)$ when $p=4$ and $n=13$. Hence we can get the result directly from Theorems 1.2 and 3.2.

In the following, we consider the case $5 \leq p \leq n-9$. First, by Lemmas 2.1 and 2.2 , we can obtain the following result.

Lemma 4.2. Suppose that $5 \leq p \leq n-9$. Then we have

$$
\begin{aligned}
& m^{+}\left(T_{1}, x\right)=C_{1} m^{+}\left(P_{n-p-4}, x\right)+C_{2} m^{+}\left(P_{n-p-5}, x\right) \\
& m^{+}\left(T_{3}, x\right)=D_{1} m^{+}\left(P_{n-p-4}, x\right)+D_{2} m^{+}\left(P_{n-p-5}, x\right)
\end{aligned}
$$

where $C_{1}=1+(p+3) x^{2}+(4 p-3) x^{4}+2(p-2) x^{6}, C_{2}=x^{2}\left[1+(p+2) x^{2}+(3 p-4) x^{4}\right]$, $D_{1}=1+(p+3) x^{2}+(4 p-2) x^{4}+(3 p-5) x^{6}$, and $D_{2}=x^{2}\left(1+4 x^{2}+3 x^{4}\right)$. 
Theorem 4.3. Suppose that $5 \leq p \leq n-9$. Then we have:

(i) If $7 \leq p \leq n-9$, then $T_{3}$ is the unique tree with third-minimal energy in $\mathbb{T}_{n, p}$.

(ii) $T_{3}$ if $n=15,17,19$ and $n \geq 21, T_{1}$ if $n=16,18,20$ is the unique tree with third-minimal energy in $\mathbb{T}_{n, 6}$.

(iii) $T_{3}$ if $n=14,16, T_{1}$ if $n=15$ and $n \geq 17$ is the unique tree with third-minimal energy in $\mathbb{T}_{n, 5}$.

Proof. By Theorems 1.2 and 3.2, we only need to compare the energies of the two graphs of $T_{1}$ and $T_{3}$. From Eq. (1), we have

$$
\begin{aligned}
E\left(T_{1}\right)-E\left(T_{3}\right) & =\frac{2}{\pi} \int_{0}^{+\infty} \frac{1}{x^{2}} \log \frac{m^{+}\left(T_{1}, x\right)}{m^{+}\left(T_{3}, x\right)} d x \\
& =\frac{2}{\pi} \int_{0}^{+\infty} \frac{1}{x^{2}} \log \left(1+\frac{m^{+}\left(T_{1}, x\right)-m^{+}\left(T_{3}, x\right)}{m^{+}\left(T_{3}, x\right)}\right) d x
\end{aligned}
$$

We consider the following cases separately:

(1) $12 \leq p \leq n-9$.

By Lemmas 2.6, 4.2 and Corollary 2.3, we have

$$
\begin{aligned}
& E\left(T_{1}\right)-E\left(T_{3}\right) \\
& \geq \frac{2}{\pi} \int_{0}^{+\infty} \frac{1}{x^{2}} \cdot \frac{m^{+}\left(T_{1}, x\right)-m^{+}\left(T_{3}, x\right)}{m^{+}\left(T_{1}, x\right)} d x \\
& =\frac{2}{\pi} \int_{0}^{+\infty} \frac{\left[(1-p) x^{4}-x^{2}\right] m^{+}\left(P_{n-p-4}, x\right)+\left[(p-2) x^{2}+(3 p-7) x^{4}\right] m^{+}\left(P_{n-p-5}, x\right)}{C_{1} m^{+}\left(P_{n-p-4}, x\right)+C_{2} m^{+}\left(P_{n-p-5}, x\right)} d x \\
& =\frac{2}{\pi} \int_{0}^{+\infty} \frac{(p-2) x^{2}+(3 p-7) x^{4}}{C_{2}+C_{1} \cdot \frac{m^{+}\left(P_{n-p-4}, x\right)}{m^{+}\left(P_{n-p-5}\right)}} d x-\frac{2}{\pi} \int_{0}^{+\infty} \frac{x^{2}+(p-1) x^{4}}{C_{1}+C_{2} \cdot \frac{m^{+}\left(P_{n-p-5}, x\right)}{m^{+}\left(P_{n-p-4}\right)}} d x \\
& \geq \frac{2}{\pi} \int_{0}^{+\infty} \frac{(p-2) x^{2}+(3 p-7) x^{4}}{C_{2}+C_{1}\left(1+x^{2}\right)} d x-\frac{2}{\pi} \int_{0}^{+\infty} \frac{x^{2}+(p-1) x^{4}}{C_{1}+C_{2} \cdot \frac{1}{1+x^{2}}} d x \\
& =\frac{2}{\pi} \int_{0}^{+\infty} \frac{(p-3) x^{2}+(2 p-7) x^{4}-(p-1) x^{6}}{1+(p+5) x^{2}+(6 p+2) x^{4}+(9 p-11) x^{6}+(2 p-4) x^{8}} d x \\
& \triangleq G(p) .
\end{aligned}
$$

For a fixed $x>0$, let

$$
g(p)=\frac{(p-3) x^{2}+(2 p-7) x^{4}-(p-1) x^{6}}{1+(p+5) x^{2}+(6 p+2) x^{4}+(9 p-11) x^{6}+(2 p-4) x^{8}} d x .
$$

Then we have

$$
g^{\prime}(p)=\frac{x^{2}+10 x^{4}+36 x^{6}+56 x^{8}+35 x^{10}+8 x^{12}+2 x^{14}}{\left[1+(p+5) x^{2}+(6 p+2) x^{4}+(9 p-11) x^{6}+(2 p-4) x^{8}\right]^{2}}>0 .
$$

So $g(p)$ is a strictly increase function of $p$. Since $G(12) \doteq \frac{2}{\pi} \cdot 0.0028451>0$, we can obtain that for $p \geq 12$,

$$
E\left(T_{1}\right)-E\left(T_{3}\right) \geq G(p) \geq G(12)>0 .
$$


(2) $p=10$ or 11 .

It follows from Eq. (5) that

$$
\begin{aligned}
& E\left(T_{1}\right)-E\left(T_{3}\right) \\
& =\frac{2}{\pi} \int_{0}^{+\infty} \frac{1}{x^{2}} \log \left(1+\frac{x^{4}\left[p-2+(3 p-7) x^{2}\right]}{D_{2}+D_{1} \cdot \frac{m^{+}\left(P_{n-p-4}, x\right)}{m^{+}\left(P_{n-p-5}, x\right)}}-\frac{x^{4}\left[1+(p-1) x^{2}\right]}{D_{1}+D_{2} \cdot \frac{m^{+}\left(P_{n-p-5}, x\right)}{m^{+}\left(P_{n-p-4}, x\right)}}\right) d x .
\end{aligned}
$$

By Corollary 2.3 and Lemma 4.2, we have

$$
\begin{aligned}
& E\left(T_{1}\right)-E\left(T_{3}\right) \\
& \geq \frac{2}{\pi} \int_{0}^{+\infty} \frac{1}{x^{2}} \log \left(1+\frac{x^{4}\left[p-2+(3 p-7) x^{2}\right]}{D_{2}+D_{1} \cdot\left(1+x^{2}\right)}-\frac{x^{4}\left[1+(p-1) x^{2}\right]}{D_{1}+D_{2} \cdot \frac{1}{1+x^{2}}}\right) d x \\
& =\frac{2}{\pi} \int_{0}^{+\infty} \frac{1}{x^{2}} \log \left(1+\frac{x^{4}\left(p-3+(2 p-7) x^{2}-(p-1) x^{4}\right)}{\left(1+x^{2}\right) \cdot\left[1+(p+4) x^{2}+(4 p+1) x^{4}+(3 p-5) x^{6}\right]}\right) d x \\
& \triangleq H(p) .
\end{aligned}
$$

By direct computation, we can obtain that $H(11) \doteq \frac{2}{\pi} \cdot 0.023681>0$ and $H(10) \doteq$ $\frac{2}{\pi} \cdot 0.0067362>0$.

(3) $p=9$.

If $n$ is even, by Eq. (6) and Lemma 2.5, we have

$$
\begin{aligned}
E\left(T_{1}\right)-E\left(T_{3}\right) & \geq \frac{2}{\pi} \int_{0}^{+\infty} \frac{1}{x^{2}} \log \left(1+\frac{x^{4}\left(7+20 x^{2}\right)}{D_{2}+D_{1} \cdot \frac{1+\sqrt{1+4 x^{2}}}{2}}-\frac{x^{4}\left(1+8 x^{2}\right)}{D_{1}+D_{2} \cdot \frac{2}{1+\sqrt{1+4 x^{2}}}}\right) d x \\
& \doteq \frac{2}{\pi} \cdot 0.11251>0 .
\end{aligned}
$$

If $n$ is odd, we want to find $n$ and $x$ satisfying that

$$
\frac{m^{+}\left(P_{n-p-5}, x\right)}{m^{+}\left(P_{n-p-4}, x\right)}>\frac{1.99}{1+\sqrt{1+4 x^{2}}} \text {. }
$$

By Lemma 2.4, it is equivalent to solve

$$
\frac{\lambda_{1}^{n-p-4}-\lambda_{2}^{n-p-4}}{\lambda_{1}^{n-p-3}-\lambda_{2}^{n-p-3}}>\frac{1.99}{2 \lambda_{1}}
$$

which means to solve

$$
\left(\frac{\lambda_{1}}{\lambda_{2}}\right)^{n-p-4}>\frac{2 \lambda_{1}-1.99 \lambda_{2}}{0.01 \lambda_{1}}
$$

that is,

$$
\left(\frac{1+\sqrt{1+4 x^{2}}}{2 x}\right)^{2(n-p-4)}>399-\frac{398}{1+\sqrt{1+4 x^{2}}}
$$


or equivalently,

$$
2(n-p-4)>\log _{\frac{1+\sqrt{1+4 x^{2}}}{2 x}}\left(399-\frac{398}{1+\sqrt{1+4 x^{2}}}\right) .
$$

Note that for $x \in(0,+\infty), \log _{\frac{1+\sqrt{1+4 x^{2}}}{2 x}}\left(399-\frac{398}{1+\sqrt{1+4 x^{2}}}\right)$ is increasing. Therefore if $x \in(0,1]$, then $\log _{\frac{1+\sqrt{1+4 x^{2}}}{2 x}}\left(399-\frac{398}{1+\sqrt{1+4 x^{2}}}\right) \leq \log _{\frac{1+\sqrt{1+4}}{2}}\left(399-\frac{398}{1+\sqrt{1+4}}\right)<12$. Thus when $n \geq 19$ and $x \in(0,1]$, Ineq. (7) holds. Hence if $n$ is odd and $n \geq 19$, from Eq. (6) and Lemma 2.5, we have

$$
\begin{aligned}
E\left(T_{1}\right)-E\left(T_{3}\right) & \geq \frac{2}{\pi} \int_{1}^{+\infty} \frac{1}{x^{2}} \log \left(1+\frac{x^{4}\left(7+20 x^{2}\right)}{D_{2}+D_{1} \cdot\left(1+x^{2}\right)}-\frac{x^{4}\left(1+8 x^{2}\right)}{D_{1}+D_{2} \cdot \frac{1}{1+x^{2}}}\right) d x \\
& +\frac{2}{\pi} \int_{0}^{1} \frac{1}{x^{2}} \log \left(1+\frac{x^{4}\left(7+20 x^{2}\right)}{D_{2}+D_{1} \cdot \frac{1+\sqrt{1+4 x^{2}}}{1.99}}-\frac{x^{4}\left(1+8 x^{2}\right)}{D_{1}+D_{2} \cdot \frac{1.99}{1+\sqrt{1+4 x^{2}}}}\right) d x, \\
& \doteq \frac{2}{\pi} \cdot\left(5.0665 \times 10^{-4}\right)>0 .
\end{aligned}
$$

(4) $p=8$.

If $n$ is odd, by Eq. (6) and Lemma 2.5, we have

$$
\begin{aligned}
E\left(T_{1}\right)-E\left(T_{3}\right) & \geq \frac{2}{\pi} \int_{0}^{+\infty} \frac{1}{x^{2}} \log \left(1+\frac{x^{4}\left(6+17 x^{2}\right)}{D_{2}+D_{1} \cdot \frac{1+\sqrt{1+4 x^{2}}}{2}}-\frac{x^{4}\left(1+7 x^{2}\right)}{D_{1}+D_{2} \cdot \frac{2}{1+\sqrt{1+4 x^{2}}}}\right) d x \\
& \doteq \frac{2}{\pi} \cdot 0.086741>0 .
\end{aligned}
$$

If $n$ is even, similar to the proof in (3), we can show that when $n \geq 22$ and $x \in(0,1.6]$, Ineq. (7) holds. Hence if $n$ is even and $n \geq 22$, from Eq. (6) and Lemma 2.5 , we have

$$
\begin{aligned}
E\left(T_{1}\right)-E\left(T_{3}\right) & \geq \frac{2}{\pi} \int_{1.6}^{+\infty} \frac{1}{x^{2}} \log \left(1+\frac{x^{4}\left(6+17 x^{2}\right)}{D_{2}+D_{1} \cdot\left(1+x^{2}\right)}-\frac{x^{4}\left(1+7 x^{2}\right)}{D_{1}+D_{2} \cdot \frac{1}{1+x^{2}}}\right) d x \\
& +\frac{2}{\pi} \int_{0}^{1.6} \frac{1}{x^{2}} \log \left(1+\frac{x^{4}\left(6+17 x^{2}\right)}{D_{2}+D_{1} \cdot \frac{1+\sqrt{1+4 x^{2}}}{1.99}}-\frac{x^{4}\left(1+7 x^{2}\right)}{D_{1}+D_{2} \cdot \frac{1.99}{1+\sqrt{1+4 x^{2}}}}\right) d x, \\
& \doteq \frac{2}{\pi} \cdot\left(5.2403 \times 10^{-4}\right)>0 .
\end{aligned}
$$

By direct computation, we get that $E\left(T_{1}\right)>E\left(T_{3}\right)$ for $n=18$ and 20 .

(5) $p=7$.

If $n$ is even, by Eq. (6) and Lemma 2.5, we have

$$
\begin{aligned}
E\left(T_{1}\right)-E\left(T_{3}\right) & \geq \frac{2}{\pi} \int_{0}^{+\infty} \frac{1}{x^{2}} \log \left(1+\frac{x^{4}\left(5+14 x^{2}\right)}{D_{2}+D_{1} \cdot \frac{1+\sqrt{1+4 x^{2}}}{2}}-\frac{x^{4}\left(1+6 x^{2}\right)}{D_{1}+D_{2} \cdot \frac{2}{1+\sqrt{1+4 x^{2}}}}\right) d x \\
& \doteq \frac{2}{\pi} \cdot 0.055170>0 .
\end{aligned}
$$


If $n$ is odd, similar to the proof in (3), we can show that when $n \geq 27$ and $x \in(0,2.6]$, Ineq. (7) holds. Hence if $n$ is odd and $n \geq 27$, from Eq. (6) and Lemma 2.5 , we have

$$
\begin{aligned}
E\left(T_{1}\right)-E\left(T_{3}\right) & \geq \frac{2}{\pi} \int_{2.6}^{+\infty} \frac{1}{x^{2}} \log \left(1+\frac{x^{4}\left(5+14 x^{2}\right)}{D_{2}+D_{1} \cdot\left(1+x^{2}\right)}-\frac{x^{4}\left(1+6 x^{2}\right)}{D_{1}+D_{2} \cdot \frac{1}{1+x^{2}}}\right) d x \\
& +\frac{2}{\pi} \int_{0}^{2.6} \frac{1}{x^{2}} \log \left(1+\frac{x^{4}\left(5+14 x^{2}\right)}{D_{2}+D_{1} \cdot \frac{1+\sqrt{1+4 x^{2}}}{1.99}}-\frac{x^{4}\left(1+6 x^{2}\right)}{D_{1}+D_{2} \cdot \frac{1.99}{1+\sqrt{1+4 x^{2}}}}\right) d x, \\
& \doteq \frac{2}{\pi} \cdot\left(1.1834 \times 10^{-3}\right)>0 .
\end{aligned}
$$

By direct computation, we get that $E\left(T_{1}\right)>E\left(T_{3}\right)$ for $n=17,19,21,23$ and 25 .

(6) $p=6$.

If $n$ is odd, by Eq. (6) and Lemma 2.5, we have

$$
\begin{aligned}
E\left(T_{1}\right)-E\left(T_{3}\right) & \geq \frac{2}{\pi} \int_{0}^{+\infty} \frac{1}{x^{2}} \log \left(1+\frac{x^{4}\left(4+11 x^{2}\right)}{D_{2}+D_{1} \cdot \frac{1+\sqrt{1+4 x^{2}}}{2}}-\frac{x^{4}\left(1+5 x^{2}\right)}{D_{1}+D_{2} \cdot \frac{2}{1+\sqrt{1+4 x^{2}}}}\right) d x \\
& \doteq \frac{2}{\pi} \cdot 0.015038>0 .
\end{aligned}
$$

If $n$ is even, similar to the proof in (3), we can show that when $n \geq 50$ and $x \in(0,6.6]$, Ineq. (7) holds. Hence if $n$ is even and $n \geq 50$, from Eq. (6) and Lemma 2.5 , we have

$$
\begin{aligned}
E\left(T_{1}\right)-E\left(T_{3}\right) & \geq \frac{2}{\pi} \int_{6.6}^{+\infty} \frac{1}{x^{2}} \log \left(1+\frac{x^{4}\left(4+11 x^{2}\right)}{D_{2}+D_{1} \cdot\left(1+x^{2}\right)}-\frac{x^{4}\left(1+5 x^{2}\right)}{D_{1}+D_{2} \cdot \frac{1}{1+x^{2}}}\right) d x \\
& +\frac{2}{\pi} \int_{0}^{6.6} \frac{1}{x^{2}} \log \left(1+\frac{x^{4}\left(4+11 x^{2}\right)}{D_{2}+D_{1} \cdot \frac{1+\sqrt{1+4 x^{2}}}{1.99}}-\frac{x^{4}\left(1+5 x^{2}\right)}{D_{1}+D_{2} \cdot \frac{1.99}{1+\sqrt{1+4 x^{2}}}}\right) d x \\
& \doteq \frac{2}{\pi} \cdot\left(8.9163 \times 10^{-5}\right)>0 .
\end{aligned}
$$

For any even $n$ with $16 \leq n \leq 48$, by direct computation, we get that $E\left(T_{1}\right)<E\left(T_{3}\right)$ for $n=16,18,20$, and $E\left(T_{1}\right)>E\left(T_{3}\right)$ for the other cases.

(7) $p=5$.

If $n$ is odd, by Eq. (6) and Lemma 2.5, we have

$$
\begin{aligned}
E\left(T_{1}\right)-E\left(T_{3}\right) & \leq \frac{2}{\pi} \int_{0}^{+\infty} \frac{1}{x^{2}} \log \left(1+\frac{x^{4}\left(3+8 x^{2}\right)}{D_{2}+D_{1} \cdot \frac{1+\sqrt{1+4 x^{2}}}{2}}-\frac{x^{4}\left(1+4 x^{2}\right)}{D_{1}+D_{2} \cdot \frac{2}{1+\sqrt{1+4 x^{2}}}}\right) d x \\
& \doteq \frac{2}{\pi} \cdot(-0.038859)<0 .
\end{aligned}
$$

If $n$ is even, similar to the proof in Theorem 3.2, we can show that when $n \geq 130$ and $x \in(0,20]$, the following inequality holds:

$$
\frac{m^{+}\left(P_{n-p-5}, x\right)}{m^{+}\left(P_{n-p-4}, x\right)}<\frac{2.01}{1+\sqrt{1+4 x^{2}}} \text {. }
$$


Hence if $n$ is even and $n \geq 130$, from Eq. (6) and Lemma 2.5, we have

$$
\begin{aligned}
E\left(T_{1}\right)-E\left(T_{3}\right) & \leq \frac{2}{\pi} \int_{20}^{+\infty} \frac{1}{x^{2}} \log \left(1+\frac{x^{4}\left(3+8 x^{2}\right)}{D_{2}+D_{1} \cdot 1}-\frac{x^{4}\left(1+4 x^{2}\right)}{D_{1}+D_{2} \cdot 1}\right) d x \\
& +\frac{2}{\pi} \int_{0}^{20} \frac{1}{x^{2}} \log \left(1+\frac{x^{4}\left(3+8 x^{2}\right)}{D_{2}+D_{1} \cdot \frac{1+\sqrt{1+4 x^{2}}}{2.01}}-\frac{x^{4}\left(1+4 x^{2}\right)}{D_{1}+D_{2} \cdot \frac{2.01}{1+\sqrt{1+4 x^{2}}}}\right) d x \\
& \doteq \frac{2}{\pi} \cdot\left(-1.4442 \times 10^{-4}\right)<0 .
\end{aligned}
$$

For any even $n$ with $14 \leq n \leq 128$, by direct computation, we get that $E\left(T_{1}\right)>E\left(T_{3}\right)$ for $n=14,16$, and $E\left(T_{1}\right)<E\left(T_{3}\right)$ for the other cases.

The proof is thus complete.

\section{Acknowledgments}

The authors would like to thank the reviewers for valuable comments and suggestions. This work was supported by National Natural Science Foundation of China (No. 11101351), Jiangsu Government Scholarship for Overseas Studies and NSF of the Jiangsu Higher Education Institutions (No. 11KJB110014).

\section{References}

[1] L. Chen, J. Liu, Y. Shi, Matching energy of unicyclic and bicyclic graphs with a given diameter, Complexity 21 (2) (2015) 224-238.

[2] L. Chen, J. Liu, Y. Shi, Bounds on the matching energy of unicyclic odd-cycle graphs, MATCH Commun. Math. Comput. Chem. 75 (2016) 315-330.

[3] L. Chen, Y. Shi, Maximal matching energy of tricyclic graphs, MATCH Commun. Math. Comput. Chem. 73 (2015) 105-119.

[4] K. C. Das , S. A. Mojallal, Relation between energy and (signless) Laplacian energy of graphs, MATCH Commun. Math. Comput. Chem. 74 (2015) 359-366.

[5] S. Drury, H. Lin, On graphs with near minimal distance energy, Linear Algebra Appl. 477 (2015) 76-101.

[6] R. Gu, X. Li, J. Liu, Note on three results on Randić energy and incidence energy, MATCH Commun. Math. Comput. Chem. 73 (2015) 61-71.

[7] I. Gutman, Acyclic systems with extremal Hückel $\pi$-electron energy of trees, Theoret. Chim. Acta. 45 (1977) 79-87.

[8] I. Gutman, The Energy of a Graph: Old and New Results, in: A. Betten, A. Kohnert, R. Laue, A. Wassermann (Eds.), Algebraic Combinatorics and Applications, Springer-Verlag, Berlin, 2001, pp. 196-211. 
[9] I. Gutman, Kragujevac trees and their energy, Sci. Publ. State Univ. Novi Pazar A 6 (2014) 71-79.

[10] I. Gutman, X. Li, J. Zhang, Graph Energy, in: M. Dehmer, F. Emmert-Streib (Eds.), Analysis of Complex Networks: From Biology to Linguistics, Wiley-VCH, Weinheim, 2009, 145-174.

[11] I. Gutman, O. E. Polansky, Mathematical Concepts in Organic Chemistry, Springer-Verlag, Berlin, 1986.

[12] B. Huo, X. Li, Y. Shi, Complete solution to a problem on the maximal energy of unicyclic bipartite graphs, Linear Algebra Appl. 434 (2011) 1370-1377.

[13] B. Huo, X. Li, Y. Shi, Complete solution to a conjecture on the maximal energy of unicyclic graphs, European J. Combin. 32 (2011) 662-673.

[14] J. Li, J. M. Guo, W. C. Shiu, A note on Randić energy, MATCH Commun. Math. Comput. Chem. 74 (2015) 389-398.

[15] J. Li, X. Li, Y. Shi, On the maximal energy tree with two maximum degree vertices, Linear Algebra Appl. 435 (2011) 2272-2284.

[16] N. Li, S. Li, On the extremal energies of trees, MATCH Commun. Math. Comput. Chem. 59 (2008) 291-314.

[17] S. Li, N. Li, On minimal energies of trees with given diameter, Electronic J. Linear Algebra 17 (2008) 414-425.

[18] X. Li, Y. Shi, I. Gutman, Graph Energy, Springer, New York, 2012.

[19] X. Li, Y. Shi, M. Wei, J. Li, On a conjecture about tricyclic graphs with maximal energy, MATCH Commun. Math. Comput. Chem. 72 (2014) 183-214.

[20] H. Ma, Some relations on the ordering of trees by minimal energies between subclasses of trees, J. Appl. Math. Comput. 45 (2014) 111-135.

[21] H. Ma, Y. Bai, S. Ji, On the minimal energy of conjugated unicyclic graphs with maximum degree at most 3, Discrete Appl. Math. 186 (2015) 186-198.

[22] C. A. Marín, J. Monsalve, J. Rada, Maximum and minimum energy trees with two and three branched vertices, MATCH Commun. Math. Comput. Chem. 74 (2015) 285-306.

[23] S. Renqian, Y. Ge, B. Huo, S. Ji, Q. Diao, On the tree with diameter 4 and maximal energy, Appl. Math. Comput. 268 (2015) 364-374.

[24] H. Shan, J. Shao, The proof of a conjecture on the comparison of the energies of trees, J. Math. Chem. 50 (2012) 2637-2647.

[25] T. Tian, W. Yan, S. Li, On the minimal energy of trees with a given number of vertices of odd degree, MATCH Commun. Math. Comput. Chem. 73 (2015) $3-10$. 
[26] W. Wang, L. Kang, Ordering of the trees by minimal energies, J. Math. Chem. 47 (2010) 937-958.

[27] W. Yan, L. Ye, On the minimal energy of trees with a given diameter, Appl. Math. Lett. 18 (2005) 1046-1052.

[28] L. Ye, X. Yuan, On the minimal energy of trees with a given number of pendent vertices, MATCH Commun. Math. Comput. Chem. 57 (2007) 193-201.

[29] A. Yu, X. Lv, Minimal energy on trees with $k$ pendent vertices, Linear Algebra Appl. 418 (2006) 625-633.

[30] B. Zhou, F. Li, On minimal energies of trees of a prescribed diameter, J. Math. Chem. 39 (2006) 465-473.

[31] V.A. Zorich, Mathematical Analysis, MCCME, 2002. 